\title{
Philosophiques
}

\section{De l'orthodoxie en philosophie}

\section{Jean-Paul Brodeur}

Volume 3, numéro 2, octobre 1976

URI : https://id.erudit.org/iderudit/203054ar

DOI : https://doi.org/10.7202/203054ar

Aller au sommaire du numéro

Éditeur(s)

Société de philosophie du Québec

ISSN

0316-2923 (imprimé)

1492-1391 (numérique)

Découvrir la revue

Citer cet article

Brodeur, J.-P. (1976). De l'orthodoxie en philosophie. Philosophiques, 3(2),

209-253. https://doi.org/10.7202/203054ar

Ce document est protégé par la loi sur le droit d'auteur. L'utilisation des services d'Érudit (y compris la reproduction) est assujettie à sa politique d'utilisation que vous pouvez consulter en ligne.

https://apropos.erudit.org/fr/usagers/politique-dutilisation/ 


\title{
DE L'ORTHODOXIE EN PHILOSOPHIE
}

\author{
(A propos de l'Académie canadienne \\ Saint-Thomas d'Aquin) \\ par Jean-Paul Brodeur
}

La philosophie, telle qu'elle s'est pratiquée au Québec, a été longtemps identifiée avec la répétition et le commentaire de thèses attribuées à saint Thomas d'Aquin. Une enquête faite par Stanley French, de l'Université Sir George Williams, a montré qu'à une date aussi tardive que 1962, plus de la moitié des professeurs de philosophie au Québec se réclamaient encore des principes du thomisme. Bien qu'il ait occupé une place considérable dans l'horizon idéologique québécois, le mouvement thomiste demeure encore relativement peu connu, une des raisons de cette lacune étant l'existence d'un sentiment diffus de honte, auquel se mêle une certaine aversion, à l'égard de cette période de notre histoire intellectuelle. $\grave{A}$ cette première raison vient s'ajouter le fait qu'on n'arrive pas très bien à imaginer l'intérêt théorique qu'il pourrait y avoir à s'employer à décrire le mouvement thomiste québécois.

Il est cependant largement temps que nous abordions sans tremblement l'histoire de notre discipline. Dans son rapport avec le passé, la philosophie québécoise s'est mise de façon croissante à ressembler au héros du roman de Charlotte Brönte, Jane Eyre, qui avait condamné l'une des ailes de son manoir parce qu'il y avait enfermé sa femme devenue folle. Bien entendu, les cris de la malheureuse se répercutaient la nuit dans toutes les pièces du manoir, remplissant ses habitants de frayeur. De la même façon, la maison de la philosophie québécoise est encore remplie des cris de tous ces prélats furieux qu'elle a enfermés dans des pièces condamnées, dont elle ne se résout plus à faire sauter les verrous. Outre ses vertus proprement thérapeutiques pour tous les habitants de la maison, la visite de ces chambres closes présente, selon moi, un intérêt théorique indéniable. 
C'est en effet la malédiction des doctrines que de s'achever sous la forme d'orthodoxies. Or si la plupart des gens s'accordent pour dénoncer les méfaits de l'instauration d'une orthodoxie, qui a pour premier résultat de stériliser la recherche en fixant d'avance les conclusions auxquelles il lui est permis de parvenir, nous possédons encore assez peu de descriptions explicites de la mise en place et du fonctionnement de ce phénomène culturel caractérisé que constitue une orthodoxie dans le domaine de la pensée. Convenons provisoirement de comprendre qu'une doctrine se meut en une orthodoxie lorsque la vérité de ses énoncés est posée comme définitive et lorsqu'il est décrété que ses thèses devront être reprises et défendues par quiconque tiendra un discours public dans le domaine qu'elle recouvre. De façon plus concise, le propre d'une orthodoxie est de redoubler le dogmatisme par une exigence absolue de conformité. Or si la mise en place d'une orthodoxie a été reconnue comme la pire des servitudes à laquelle pouvait être soumise la pensée, qui ne voit pas l'intérêt de fournir une description exacte de ce processus afin de pouvoir en reconnaître les premiers stades et de conjurer son accomplissement?

Le thomisme québécois a constitué le paradigme de ce que l'on pourrait s'entendre à dénommer une orthodoxie. Je poursuivrai dans ce texte' une triple tâche. Je tenterai d'abord, bien que de façon restreinte, de faire ouvre d'historien en présentant une description textuelle d'un fragment de cette partie de notre histoire intellectuelle qu'a constitué le thomisme. Cette première description me fournira la matière d'une élaboration proprement théorique par laquelle j'essaierai d'identifier de façon formelle les divers traits qui caractérisent le discours orthodoxe en lui-même. C'est cette élaboration théorique qui constitue l'objet principal de ce

1. Ce texte a déjà été présenté sous forme de conférence publique au Cegep de Sherbrooke pendant l'année académique 1975-76. A la suite de remarques de divers collègues, plusieurs modifications ont été apportées au texte original de la conférence. Je tiens à cet égard à remarquer plus particulièrement Yvan Lamonde, Georges Leroux et Robert Nadeau.' Il va de soi que j'assume seul la responsabilité des imperfections qui demeurent dans mon texte. Les deux remarques finales sont le résultat partiel de discussions très fécondes qui se sont tenues dans un séminaire de maîtrise du Département de Philosophie de l'Université du Québec à Montréal pendant le semestre d'hiver 1976. Denis Charlebois, Pierre Coupal, Claude Gervais, Marc Leclerc, André Pelletier et Normand Piché ont participé à ce séminaire. Qu'ils soient remerciés de leur contribution. 
texte. J'estime enfin qu'en m'employant à réaliser les deux tâches précédentes, je contribuerai à ma façon à nous rendre plus conscients des divers facteurs qui ont concouru à produire le contexte historique dans lequel nous sommes actuellement plongés.

1 - Le thomisme québécois a constitué un très long épisode de l'histoire de notre activité philosophique, Il n'en est aucun qui, au point de vue de son importance, puisse lui être comparé. Aussi convient-il que d'entrée de jeu, je fixe la matière et les limites de cette étude, dont l'objet ne recoupe pas la totalité de l'histoire du thomisme au Québec. De façon plus précise, la matière de mon étude a été exclusivement puisée dans onze volumes, récemment acquis par la bibliothèque de l'Université du Québec à Montréal (UQAM), où sont reproduits les textes des conférences qui ont été prononcées dans le cadre des séances annuelles de l'Académie canadienne Saint-Thomas d'Aquin ${ }^{2}$. Bien que ma connaissance du thomisme québécois soit loin d'être complète, je ne crois pas qu'il y ait eu une époque de son développement où il ait été plus influent que pendant l'existence de cette Académie canadienne-française Saint-Thomas d'Aquin, sur laquelle il convient maintenant de donner quelques renseignements.

L'Académie canadienne Saint-Thomas d'Aquin a été fondée le 16 janvier 1930 par le cardinal Rouleau et son premier président fut Monseigneur Louis-Adolphe Pâquet. Monseigneur Pâquet devait rester le président et l'animateur infatigable de cette Académie jusqu'en 1940, date à laquelle le chanoine Gagnon le remplaça. La collection de volumes achetés par la bibliothèque de

2. Voici la référence exacte de ces volumes. L'Académie canadienne Saint-Thomas d'Aluin. Fondation. Première Session (12.13 novembre 1930), Québec. Typ. "LAction Catholique", 1932. Ce titre réfère au premier volume des travaux de l'Académie canadienne Saint-Thomas d'Aquin. Les autres volumes ont le même titre que le premier et ont été publiés à Québec, par les soins de l'Action Catholique (à l'exception du dernier volume que nous avons consulté). Voici la date de ces publications: 1933 (Seconde Session, 21.22 octobre 1931); 1934 (Troisième Session, 12-13 octobre 1932); 1935 (Quatrième Sesssion, 4.5 octobre 1933); 1936 (Cinquième Session, 17-18 octobre 1934); 1937 (Sixième Session, 9.10 octobre 1935); 1938 (Septième Session, $7-8$ octobre 1936) ; 1939 (Huitième Session. 13.14 octobre 1937); 1911 (Neuvième Session. 5.6 octobre 1938): 1912 (Dixième Session, 23-2.1 octobre 10.10) et Onzième Session. 15.16 actobre 1941 ) ; 1945 (Douxième Session, 14-15 octobre 1942 et Treizième Session, 20 )-21 octobre 1943). Le dernier volume cité - celui de 1945 - a été publié par l'Imprimerie Franciscaine et ne porte pas, comme tous les autres, la mention " Action Ca. tholique $"$. 
l'UQAM s'arrête à la reproduction des textes présentés à la treizième session, qui se tint les 20 et 21 octobre 1943 . Je ne sais si l'Académie se réunit à nouveau au-delà de $1943^{3}$. Quoi qu'il en soit de cette question, l'ensemble des volumes que nous possédons et qui couvre la période qui s'étend de la fondation de l'Académie en 1930 à l'année 1943, avec l'exception de l'année 1939, où l'Académie suspendit sa réunion annuelle, est assez important pour être considéré comme significatif. Je me permettrai, au cours de ce texte, de citer abondamment les actes de l'Académie canadienne Saint-Thomas d'Aquin. Pour deux raisons. 1) D'abord, parce que ces textes sont maintenant très peu connus des jeunes chercheurs et que leur contenu a été oubiíe par les autres. 2) Ensuite, parce que certaines des affirmations qu'ils contiennent sont, à certains égards, tellement stupéfiantes qu'il m'est souvent apparu nécessaire de les produire sous forme de citations afin de me prémunir à l'avance du reproche d'en caricaturer le contenu.

L'Académie canadienne Saint-Thomas d'Aquin a d'abord été composée de vingt-neuf membres. Ce nombre ne pouvait excéder trente. Ses membres étaient dans leur très grande majorité des clercs : je ne pense pas qu'il y ait jamais eu, de 1930 à 1943, plus de quatre laïques sur la trentaine de membres constituant l'Académie. Ce nombre a été le plus souvent restreint à trois. À la trentaine de membres de l'Académie s'est bientôt adjoint un comité composé d'un président d'honneur, l'archevêque de Québec, et de plusieurs vice-présidents d'honneur où figuraient les divers recteurs des universités francophones (Laval, Montréal et Ottawa) et le

3. J'ai fait des recherches dans les bibliothèques des Universités Laval, d'Ottawa, de Montréal et à celle de l'Institut d'Études Médiévales de Montréal. À part celle de l'Université de Montréal, qui ne semble pas posséder les volumes publiés par l'A. cadémie canadienne Saint-Thomas d'Aquin (aucune carte au fichier), toutes les bibliothèques où je suis allé possèdent une collection de ces volumes qui, comme celle de l'Université du Québec à Montréal, s'arrête à la session de 1943 (la treizième). Les archives de l'Université d'Ottawa possèdent un exemplaire du programme des communications présentées à la quinzième session de l'Académie ; cette session s'est tenue à l'Université d'Ottawa, les 3 et 4 novembre 1945, sous la présidence de l'archevêque d'Ottawa, Monseigneur A. Vachon. Cette quinzième session a dû être précédée d'une quatorzième session, en 1944. Je n'ai pas trouvé d'autres indications de la poursuite publique des travaux de l'Académie canadienne Saint-Thomas d'Aquin au-delà de 1945. Je n'ai pas, non plus, trouvé de volumes colligeant les textes des communications présentées aux sessions de 1944 et de 1945. Des témoins de cette époque pourront sans doute nous éclairer sur le sort de l'Académie thomiste après 1945. Je ne connais malheureusement aucune de ces personnes. 
Provincial des Dominicains. Cette liste n'est pas exhaustive. Les réunions se tenaient une fois l'an, pendant deux jours, et divers conférenciers, choisis la plupart du temps parmi les membres de l'Académie - il y eut cependant un nombre considérable d'exceptions à cette règle - y lisaient un texte écrit sur un sujet déterminé à l'avance par Mgr Pâquet. Le parterre devant lequel ces conférences se prononçaient était extrêmement prestigieux, pour ce qui est du Québec des années trente. Qu'on en juge : la séance se déroulait devant les membres de l'Académie Saint-Thomas euxmêmes. Or, à cette époque où les sciences expérimentales étaient décriées et les sciences sociales encore assez peu existantes au Québec, les membres de l'Académie Saint-Thomas d'Aquin, appartenant aux Facultés de Théologie, de Philosophie et de Droit du monde francophone, en représentaient une façon d'élite intellectuelle. À ceux-ci s'ajoutaient les vice-présidents d'honneur, c'est-à-dire, comme nous venons de le voir, les recteurs des universités francophones, et un nombre considérable d'évêques, parmi lesquels se trouvait le président d'honneur de la session, invité à la fin à féliciter les conférenciers de la journée. Au-dessus de tout ce monde trônaient, d'une part, le pouvoir politique, représenté par le premier ministre de la province de Québec, assorti du LieutenantGouverneur de la province, et, d'autre part, le pouvoir ecclésiastique représenté par le cardinal en fonction - - ce fut, pour toute cette période, le cardinal Villeneuve, le cardinal Rouleau étant décédé peu de temps après la fondation de l'Académie - et le délégué apostolique de Rome. On peut affirmer, sans grande crainte de se tromper, qu'il n'est pas d'association universitaire québécoise qui soit parvenue, depuis la disparition de l'Académie Saint-Thomas d'Aquin, à tenir ses journées annuelles devant une assemblée aussi influente.

Les textes qu'on lisait devant cette assemblée peuvent être regroupés en quatre catégories : 1) Des exposés de la pensée de saint Thomas sur des points de doctrine philosophique ou théologique relativement scolaires. Par exemple: la théorie de l'acte et de la puissance chez saint Thomas. 2) Des textes qui, de façon explicite ou voilée, se rapportaient à des problèmes de nature sociale ou politique. Par exemple: un texte sur les juridictions respectives du fédéral et du provincial en matière d'éducation. 3) D'autres textes ont comparé la doctrine de saint Thomas avec celle d'autres penseurs, parmi lesquels le plus souvent cité est saint Augustin. 4) 
Enfin des commandes, souvent lues en anglais, et où la communauté francophone tentait de prendre connaissance de ce qui se faisait dans divers secteurs de l'éducation et de la pensée anglophones. Par exemple : l'enseignement de la théologie dans les facultés protestantes.

Lorsqu'ils étaient publiés, ces textes jouissaient d'une diffusion assez large dans des académies régionales qui étaient rattachées à la grande Académie canadienne Saint-Thomas d'Aquin et assuraient la croissance de son influence. De telles académies régionales, qui faisaient profession de suivre les mêmes principes que l'Académie canadienne originaire, existaient à Chicoutimi, Montréal et Ottawa. Il a dû y en avoir d'autres.

Il est une dernière remarque que j'aimerais faire avant de passer à l'analyse des textes qui sont reproduits dans les Actes de cette Académie. Il n'y a pas lieu de trop s'étonner du nombre de clercs au sein de l'Académie Saint Thomas d'Aquin. L'enseignement universitaire, et même collégial de la philosophie, était à cette époque presque entièrement assuré par des clercs. Cette situa. tion n'était pas propre au Canada français. C'étaient en grande partie des ministres protestants qui assuraient l'enseignement de la philosophie du côté anglophone. La proportion des clercs au sein de l'Académie canadienne Saint-Thomas d'Aquin - neuf clercs pour dix membres - donne une image assez fidèle de la proportion entre les clercs et les laïques qui existait dans l'enseignement. On a trop tendance à oublier cette réalité cléricale de l'enseignement de la philosophie au Québec. Cette réalité continue d'opérer même aujourd'hui, comme le montrerait un examen de la composition des divers Départements de Philosophie francophones. La proportion de ceux qui ont, à un moment ou un autre de leur carrière, occupé une place au sein de l'appareil clérical demeure considérable.

2 - J'en viens maintenant à l'analyse des textes produits par l'Académie Saint-Thomas. Voici quelle est la procédure que je suivrai. Je développerai cette analyse en présentant un certain nombre de thèses sur la nature d'une orthodoxie en philosophie. J'illustrerai chaque fois ces thèses à l'aide d'un exemple pris dans le corpus des textes dont je viens de décrire sommairement la provenance. Cette prodécure laisserait entendre que les textes thomistes n'ont dans cet exposé que la fonction d'illustrer une théorie géné- 
rale de l'orthodoxie qui aurait été produite indépendamment d'eux. Il y a là une fiction dont il faut révéler l'existence, pour dissiper des malentendus. Le texte thomiste ne constitue pas pour l'instant un cas particulier de la théorie de l'orthodoxie que je vais élaborer dans ce texte. Au contraire, cette théorie n'est qu'une généralisation des propriétés que j'ai découvertes en faisant l'analyse des seuls textes produits par l'Académie Saint-Thomas d'Aquin. Je n'ai pas encore mis à l'épreuve la théorie que j'ai construite à la suite de ma lecture des Actes de l'Académie canadienne Saint-Thomas, en examinant si elle pouvait rendre compte d'autres orthodoxies que le thomisme en philosophie. J'entreprendrai cet examen en un autre lieu ${ }^{4}$. Je n'ai cependant pas cru devoir renoncer à présenter sous forme de thèses générales sur l'orthodoxie des résultats qui ont été obtenus à la suite de la description d'un seul type d'orthodoxie. Les mérites didactiques de ce mode d'exposition m'apparaissent considérables, si l'on dissipe au départ, comme je viens de le faire, les malentendus qu'il pourrait engendrer. Les thèses que je vous présente sous une forme un peu dogmatique ne sont donc, en réalité, que des hypothèses de recherche obtenues à la suite d'un travail sur un seul ensemble de textes - ceux de l'Académie Saint-Thomas - et leur fécondité devra se tirer de leur puissance à rendre compte d'autres types d'orthodoxies que celle dont il sera question dans la suite de cet exposé. Je ne doute pas cependant que mes hypothèses résisteront à cette épreuve. Ceci dit, j'énoncerai maintenant mes thèses.

\section{$2.1-$}

THĖSE NO 1

Une orthodoxie constitue essentiellement un phénomène institutionnel. Exprimée de façon plus précise, une doctrine ne devient une orthodoxie qu'à la condition de voir sa vérité instituée par une instance qui possède un pouvoir de contrainte efficace sur ceux à qui elle veut imposer cette doctrine.

La raison de cette première thèse est obvie. On sait qu'il est dans les diverses sciences certaines propositions qui sont reçues comme vraies par la communauté des chercheurs et que l'on ne saurait nier sans heurter un consensus. Il est impérieux que l'on

4. Il me faut dire que quelques chercheurs qui ont lu ce texte avant sa publication et qui travaillent sur des corpus différents du mien m'ont affirmé que plusieurs de mes thèses s'appliquaient aux textes qu'ils analysent. 
évite de confondre ce qui constitue un paradigme scientifique, l'état des connaissances dont la vérité est reconnue à une époque donnée du développement d'une science - et qui n'est qu'un résultat normal de la recherche scientifique, avec l'instauration d'une orthodoxie, qui appartient de façon irrémédiable à la pathologie du travail intellectuel. Si un savant ne saurait mettre en question avec légèreté les équations de la mécanique quantique, il lui est néanmoins permis de poursuivre des recherches qui mèneraient éventuellement à la modification de ces équations et de présenter le résultat de ses travaux sans se voir exclure, sauf dans des cas qui deviennent de plus en plus rares, de l'université où il enseigne et, de façon plus large, de la communauté des chercheurs.

Il n'en est malheureusement pas ainsi dans le cas de ce qui constitue une orthodoxie véritable. Le désaccord est alors habituellement sanctionné par un ostracisme ou par quelque mesure encore plus extrême. La doctrine de saint Thomas a été officiellement reconnue comme étant celle qui devait régler les positions de l'Église et des universités catholiques en matière de théologie et de philosophie par une encyclique de Léon XIII (Æterni Patris, 1879). Sa vérité a donc été officiellement instituée par l'Église, qui était une instance disposant alors d'un pouvoir de contrainte effectif sur ses membres. Le désaveu des thèses de saint Thomas d'Aquin aurait, dans le cas d'un professeur enseignant dans une institution catholique, entraîné un rappel à l'ordre énergique et éventuellement, s'il avait persisté, la mise au ban de cette institution.

Une orthodoxie est donc une doctrine dont la vérité est instituée par une instance. Cette instance est, dans la grande majorité des cas, de nature religieuse ou politique. Il est en effet rare que cette instance soit elle-même de nature scientifique. La raison en est simple : une instance d'ordre scientifique ne saurait exiger une doctrine en orthodoxie sans renoncer en même temps à son caractère scientifique. Il suit de ces remarques qu'une orthodoxie doit être caractérisée par sa double appartenance. En tant que doctrine, elle appartient au domaine des productions théoriques ; en tant que doctrine instituée, elle relève de l'instance, religieuse ou politique, qui l'a instituée.

Les conséquences de cette double appartenance sont nombreuses. Celle dont la portée est la plus considérable est la sui- 
vante: parce qu'une orthodoxie est liée par une appartenance double, son contenu est un corps mixte où les prescriptions de l'instance responsable de son institution s'efforcent toujours de subvertir le contenu doctrinal originaire, quand il ne sert pas de façon assez immédiate les intérêts de cette instance. Ces prescriptions viennent de façon voilée s'intégrer à la doctrine et participent dès lors de son caractère universel. L'instauration d'une orthodoxie rend donc possible, et ceci est important, deux opérations de nature converse : ce qui était originalement une doctrine devient par le moyen de son institution une prescription - tous les esprits devaient en effet se soumettre à cette doctrine - et ce qui dans le futur ne serait apparu que comme une prescription relativement arbitraire - des directives de l'autorité politique ou ecclésiastique sur telle ou telle matière - se verra assimilé à la doctrine. Essayons d'illustrer tout cela.

Double appartenance du thomisme, d'abord. Je me bornerai à vous citer deux textes qui sont particulièrement éloquents à cet égard. Dans l'introduction du premier volume reproduisant les travaux de l'Académie, Mgr Pâquet écrit :

Texte 1:

Selon la lettre et l'esprit des statuts académiques, les travaux présentés à chaque session ont offert un double caractère : attacbement inviolable aux principes thomistes si hautement préconisés par l'Église ; souci d'aborder et de résoudre à la lumière de ces principes, les plus graves problèmes de doctrine religieuse, philosophique et sociale, de l'époque actuelle. (Nous avons souligné).

L. - A. Pâquet, Première session, p. IV.

Notons qu'il est dans cette introduction avant tout question des principes du thomisme lui-même. La lettre par laquelle le secrétaire d'État du Vatican, le cardinal Pacelli (futur Pie XII), donne son accréditation à la nouvelle société jette cependant une autre lumière sur les raisons de sa fondation :

Texte 2 :

Le Saint Père a appris avec une vive satisfaction que votre Éminence a fondé naguère l'Académie canadienne Saint-Thomas d'Aquin, à l'instar de l'Académie romaine du même nom, dans le but de faire mieux connaître au Canada les directives du SaintSiège en matière de philosophie et de théologie.

E. Pacelli, Première session, p. 15-16. 
Il n'est question dans ce texte que du Saint-Siège et de ses directives. Le thomisme relève d'une première façon de la raison théorique qui se déploie dans les Sommes de saint Thomas, mais il relève aussi et dans une mesure égale de la raison ecclésiale qui émane du Saint-Siège.

Cette double appartenance détermine l'ambivalence du contenu des textes produits par l'Académie. Comme le laisse entendre Mgr Pâquet, les travaux de l'Académie seront d'une double nature. Ils essaieront d'abord de déterminer quelle est, sur divers points, la lettre des principes thomistes. Ces efforts produiront des énoncés de doctrine assez scolaires. Dans un second type de travaux, on s'efforcera d'éclairer les problèmes contemporains les plus pressants à la lumière des principes analysés précédemment. Or, contrairement au vœu de Mgr Pâquet, ces deux grands types de travaux - énoncés de doctrine fondamentale et application des principes - n'utilisent pas les mêmes matériaux théoriques. Si les premiers se réfèrent nécessairement à la doctrine de saint Thomas, les seconds utilisent presque exclusivement les directives exprimées dans les encycliques papales. Le texte du père Archambault sur "La restauration de l'ordre social " (Deuxième session) porte, de façon significative, en sous-titre: "d'après les encycliques Rerum Novarum et Quadragesimo Anno ". L'encyclique vient relayer la doctrine thomiste dans presque tous les cas où il est davantage question d'un problème social concret que de l'exégèse explicite des cuvres de saint Thomas. Comme ces textes plus insérés dans la réalité sociale constituent, avec les énoncés de doctrine, l'ensemble qui revendique le plus grand nombre de pages dans les Actes de l'Académie, il s'ensuit que la place que tiennent dans les Actes les encycliques est aussi considérable que celle des Sommes de saint Thomas proprement dites.

On aurait tort d'y voir une contradiction. Cette pratique apparaît pleinement justifiée dès le moment où nous rappelons à notre mémoire ce qui a été dit précédemment de la double opération que rend possible la mise en place d'une orthodoxie : la doctrine et l'institution qui la promulgue se renforcent l'une l'autre selon une logique parfaitement circulaire. Parce que le thomisme a été déclaré la doctrine officielle de l’Église, ses principes doivent être reconnus par tous les croyants. C'est là la première opération qui fait d'une doctrine une prescription. Mais parce que les paroles 
du Saint-Siège constituent en elles-mêmes la doctrine de l'Église, tout ce qui émane du Saint-Siège ne saurait être à son tour que thomiste. C'est là la seconde opération, qui confere le statut d'une doctrine à des directives circonstanciées. Ainsi se trouve annulé l'écart qui sépare la raison théorique, qui a le pouvoir de décréter ce qui est vrai, et la raison ecclésiale qui perçoit ce qui est avantageux. Le théorique s'incarne historiquement en prenant le visage d'un conformisme et le politique assume la figure du nécessaire en se donnant pour une science.

\section{$2.2-$}

THËSE NO 2 :

L'institutionnalisation d'une pensée, quel que soit son contenu, ressortit à ce que nous désignerons comme un idéalisme pratique. Ce type d'idéalisme est articulé par les catégories de l'éristique.

Cette thèse formule une remarque qui frôle en réalité le sens commun. Convenons d'abord d'appeler idéaliste une position qui privilégie le pouvoir de la conscience sur la force des choses, et réaliste ou matérialiste une position qui inverse ce privilège. La première partie de ma thèse tient dans l'affirmation suivante : même si le contenu d'une doctrine répudie de façon explicite la position idéaliste, le fait même que l'on s'estime contraint de transformer cette doctrine en une orthodoxie équivaut, en lui-même, à une reconnaissance pratique de cette position et à un acquiescement tacite à sa vérité. Pourquoi, en effet, met-on en place une orthodoxie? Essentiellement, c'est pour préserver de l'erreur ceux à qui on ne reconnaît pas le pouvoir de départager par eux-mêmes le vrai du faux. Mais l'importance d'avoir des idées justes est d'autant moindre que l'on estime que l'action humaine ou l'histoire sont entièrement déterminées par des forces matérielles - la force des choses. La crainte qui détermine une instance politique ou religieuse à désigner explicitement une doctrine comme orthodoxe est très précisément l'aveu que le pouvoir de la conscience déborde la somme des déterminismes qui s'exercent sur l'action humaine. On ne l'a pas assez remarqué, le pouvoir qui est effectivement reconnu par quelqu'un à la conscience peut être exactement mesuré par la force qu'il attribue aux idées fausses. Cette force doit être considérable pour conduire à la mise en place d'une orthodoxie avec tout son appareil de censeurs ; cette mise en place constitue donc une reconnaissance à rebours ou par l'absurde du fond d'une position 
de type idéaliste. Quelle qu'ait été la répugnance théorique maintes fois exprimée du réalisme thomiste en tant que mouvement idéologique pour toute forme d'idéalisme, ce thomisme n'a jamais cessé d'être obsédé par la puissance effective de l'idée sur le réel. Entendons ici par le réel surtout la réalité historique ; on apercevra mieux de cette façon pourquoi dans la formulation de la seconde thèse le qualificatif de pratique est attribué au terme d'idéalisme.

On peut donner de très nombreux exemples de cette terreur de l'idée fausse à laquelle nous venons de nous référer. Je me contenterai de deux textes.

Texte 3:

Une seule déviation de la pensée dans la question très ancienne, et jamais vieillie, de la connaissance suffit pour rompre l'équilibre du monde, pour ébranler les bases de la croyance, pour supprimer Dieu.

$$
\text { L. -A. Pâquet, Première session, p. } 27 .
$$

Texte 4:

Or le concile, remarque le grand historien Mœehler, et $j$ 'attire votre attention sur ce point, est à tendances nettement positives; volontairement, il exclut le côté spéculatif de la théologie.

La raison de cette préférence : le danger. (Nous soulignons). Nous savons en effet qu'en temps de crise les défenseurs de la foi, suscités par Dieu, s'en tiennent à la théologie positive...

H. Mathiot, Neuvième session, p. 33.

Ces deux textes peuvent nous introduire à la seconde partie de la deuxième thèse que je vous ai soumise. Le terme d'éristique que j'ai employé vient du grec "eris " qui signifie lutte ou combat et désigne la théorie des débats ou l'art de la controverse. Lorsque j'ai affirmé que le type d'idéalisme auquel se rattachait l'établissement d'une orthodoxie était articulé par les catégories de l'éristique, je faisais référence à l'introduction dans la littérature qui exprime la pensée orthodoxe de toutes ces métaphores qui gravitent autour des notions de lutte et d'agression. L'orthodoxie est habituellement décrite comme étant une doctrine qui nous préserve des ténèbres de "l'erreur" ou de la "déviation"; sa vérité est constamment assiégée par des "ennemis" qui s'emploient à faire tomber les fidèles dans des embûches et des pièges ; leur seule motivation est "la haine " et il faut mener contre eux une "lutte " aussi vigou- 
reuse qu'incessante. L'un des effets les plus nocifs de l'instauration d'une orthodoxie est de dramatiser, le plus souvent de façon loufoque, le processus de la connaissance. Cette dramatisation du processus de la connaissance est loin d'être aussi anecdotique qu'on a accoutumé de le penser. Ses effets sont doubles. Elle constitue d'abord un alibi global qui permet que les travaux théoriques les plus abstrus et les plus parfaitement inutiles soient conçus comme des interventions opportunes dans la grande guerre des discours que se livrent les forces du bien et du mal. Il n'est dès lors pas un seul texte qui soit suffisamment distant de la réalité sociale pour être privé des prestiges du militantisme. Mais en plus de fournir une caution à des pratiques parfaitement déréalisées, une dramatisation du travail intellectuel a pour second effet de transformer en des affrontements entre les personnes ou entre les institutions toute forme de désaccord théorique. La discussion et la critique qui constituent la vie même de la recherche cessent bientôt d'exister, la plupart des chercheurs trouvant plus économique de paraître consentir que de s'engager dans des querelles interminables.

\section{$2.3-$}

THÈSE NO 3: Une orthodoxie constitue une formation réactionnelle.

Comme la précédente, cette thèse comprend dans sa formulation des expressions qu'il nous appartient de définir. Avant de fournir l'esquisse de cette définition, j'aimerais faire état des divers motifs qui m'ont conduit à utiliser cette expression de formation réactionnelle.

L'un des traits les plus marqués du texte de l'orthodoxie est sa systématique incohérence. Cette incohérence, qui se manifeste par de nombreuses contradictions dans les textes thomistes que nous avons analysés, prend le plus souvent la forme d'une incomptabilité entre les effets désirés de la mise en place d'une orthodoxie et ses effets réels ou encore entre la théorie qu'une orthodoxie fait d'elle-même et sa pratique. Donnons d'abord quelques exemples des contradictions qui font constamment éclater le texte thomiste. Nos exemples seront au nombre de quatre et ils se suivront selon un degré de généralité croissante. 
Premier exemple

Dans un texte qui commente la méthode de saint Thomas d'Aquin, l'abbé Joseph Ferland nous apprend que saint Thomas n'est pas traditionaliste et qu'en thomisme l'argument d'autorité constitue le plus faible de tous les arguments. Le texte poursuit évidemment en recommandant aux fidèles de suivre l'exemple de saint Thomas d'Aquin. C'est déjà une contradiction bénigne que d'appuyer sur un argument d'autorité - l'exemple du maître la prescription d'y voir un mode inférieur de la démonstration. Mais à cette première inconsistance s'ajoute, cette fois de façon absolument massive, les innombrables exemples que l'on pourrait citer et où le texte des thomistes a recours à l'argument d'autorité. Ces exemples innombrables ne font que se révéler conformes à la loi qui ordonne la raison orthodoxe : lorsque la vérité d'une doctrine est officiellement instituée, le seul mode d'argumentation véritablement légitime est celui qui invoque son autorité. Il était dès lors parfaitement inconséquent de s'affirmer thomiste, par conformité avec les prescriptions des encycliques, et de déclarer dans un même souffle son peu d'estime pour l'argument d'autorité. L'acceptation de la doctrine de saint Thomas est en elle-même soumission à l'autorité.

\section{Second exemple}

Mon second exemple est de portée plus générale. Je me contenterai d'abord de vous lire ces deux textes du Cardinal Villeneuve :

Texte 5:

$$
\begin{aligned}
& \text { Peut-être le prestige et la valeur objective de notre } \\
& \text { enseignement philosophique nous ont-ils trop vite } \\
& \text { satisfaits. On a beaucoup vécu de répéter les doc- } \\
& \text { teurs. On a trop peu imité le labeur qui fouille. On } \\
& \text { n'a pas beaucoup construit de majestueuses syn- } \\
& \text { thèses personnelles propres aux exprits puissants. } \\
& \text { Mgr. J.-M.-R. Villeneuve, Première session, } \\
& \text { p. } 242 \text {. }
\end{aligned}
$$

La requête de celui qui allait devenir le cardinal Villeneuve semble claire : la philosophie thomiste québécoise doit être originale. Mais comment réclamer l'originalité de ceux-là mêmes à qui on avait déclaré quelques pages avant : 
Texte 6 :

S'il est un point acquis dans la discipline doctrinale actuelle du catholicisme, c'est sans conteste la dictature intellectuelle de l'Angélique docteur. "Ceux qui veulent être vraiment philosophes (et les religieux doivent surtout le vouloir) sont obligés ", écrivait Léon XIII le 25 novembre 1898, aux Frères mineurs, "d'établir les principes et les bases de leur doctrine sur saint Thomas d'Aquin ". "S'il se rencontre des docteurs en désaccord avec saint Thomas " avait-il précédemment exposé aux jésuites (30 décembre 1892) "quel que soit par ailleurs leur mérite, l'hésitation n'est pas souhaitable, les premiers doivent être sacrifiés au second ". (Nous avons souligné).

Mgr. J.-M.-R. Villeneuve, Première session, p. $210-211$.

La contradiction est ici tellement dure qu'il est à peine besoin de commenter : c'est vouloir casser les intelligences que de les. inviter à conserver leur liberté de réflexion tout en exigeant leur soumission à ce qui est explicitement désigné comme une dictature.

\section{Troisième exemple}

Nos deux premiers exemples, selon des degrés divers de généralité, concernent une délégation par le texte orthodoxe de son rapport réel avec la doctrine instituée : on prétend, d'une part, mépriser l'argument d'autorité alors qu'il est le seul qui, dans la pratique, soit effectivement utilisé ; on requiert, d'autre part, l'originalité tout en exigeant la soumission. Nos deux autres exemples porteront le débat encore plus haut. Nous procéderons comme dans le cas précédent en juxtaposant des textes contradictoire. Les deux textes que nous regarderons maintenant ensemble sont tirés d'une communication qui confronte les mérites respectifs des deux grandes doctrines catholiques rivales, celle de saint Thomas et celle de saint Augustin. Bien entendu, saint Thomas est le plus grand des deux. La raison de sa supériorité est intéressante : saint Augustin, entièrement soumis aux prescriptions de sa foi, n'est en effet pas reconnu comme un philosophe véritable:

Texte 7 :

En nous tenant à ces cadres rigides, peut-on parler de philosophie augustinienne? Personne n'y songe. L'augustinisme n'ayant jamais voulu être autre chose qu'une exploration rationnelle du contenu de 
la foi a perdu tout droit à s'appeler une philosophie au sens thomiste du mot. Mais est-ce tout de même une yéritable philosophie? Nous ne le pensons pas. M.-C. Forest, Première session, p. 55.

Il semblerait donc, d'après ce texte, que l'acte de philosopher doive prendre une nécessaire distance avec les contenus de la foi et de façon plus générale avec l'instance religieuse. Or, encore ici, il n'y a qu'une apparence. Écoutons parler le père Forest, quelques pages plus loin :

Texte 8 :

Si on peut prier par le cœur de saint Thomas, c'est pas son intelligence qu'il faut penser, Mgr Paquet, dans le magnifique discours-programme que nous venons d'entendre, en a donné de multiples raisons. Une seule suffirait : la volonté de l'Église. (...) Il n'est pas question de préférences personnelles, mais de soumission et d'obéissance.

M.-C. Forest, Première session, p. $69-70$

Saint Augustin, en réfléchissant les contenus de sa foi, n'est sûrement pas plus lié que le philosophe thomiste qui embrasse la doctrine de son maître pour se plier à la volonté de l'Église. On pourrait même penser qu'il est moins compromettant pour un philosophe de se reconnaître dépendant du contenu d'une foi que des décrets d'une Église. Pourquoi alors refuser à Augustin le titre de philosophe tout en s'obstinant à parler de Philosophie thomiste?

\section{Quatrième exemple}

Cette dénégation de la subordination de la philosophie à l'autorité religieuse atteint, je crois, son point culminant dans un texte de l'abbé Arthur Robert sur la philosophie du communisme. Citons-en, encore une fois, un extrait :

Texte 9:
Et comme l'ont fait les positivistes en métaphysique, Karl Marx en économie a, pour ainsi dire, substitué la croyance à la science. Ses prédictions, l'important est d'y croire. Ainsi le marxisme devient une religion, ou mieux une dévotion, un objet de foi. Un vague mysticisme, une sorte de culte vient comme pallier les contradictions qui surgissent de toutes parts et relèguent au dernier plan des éléments scientifiques et objectifs.

A. Robert, Cinquième session, p. 78-79. 
Ce texte, il faut l'avouer, est particulièrement saisissant puisqu'on y voit un abbé prendre le parti de la science contre celui du mysticisme et surtout du religieux! Soulignons-le avec force : le reproche qui est ici fait à Marx est précisément d'avoir produit une théorie dont les connotations étaient religieuses. Or, cette prise de position en faveur de la raison objective n'empêchera nullement le thomisme de prendre le parti du mystère contre la science, lorsque l'on pensera qu'il est opportun de le faire, comme en témoigne ce texte de Hermas Bastien, dont le titre est "Le mystère de la connaissance "(Neuvième session). Le terme de formation réactionnelle que nous avons précédemment utilisé pourra, à la lumière de ces exemples, être expliqué avec plus d'aisance. Ce terme désigne dans la psychologie freudienne les efforts que fournit un individu pour assumer une personnalité contraire à ses tendances profondes, et dont les traits lui sont le plus souvent dictés par ce qu'il croit être les attentes de son milieu. Ainsi, pour prendre des exemples simples, un individu lubrique se contraindra au scrupule s'il vit dans une société puritaine; de la même façon, un libre penseur se prendra pour un dévôt s'il est inséré dans une société cléricale. Naturellement, ces tentatives pour assumer une personnalité conforme à ce qui est attendu ne réussissent aux mieux qu'à engendrer des névroses, le refoulé parvenant toujours à se manifester sous une forme ou sous une autre.

Or, tel est bien ce qui semble se passer au sein du mouvement thomiste québécois. Alignons d'abord les foyers de tension. 1) Bien qu'il affecte mépriser l'argument d'autorité, le thomisme constitue en lui-même une structure d'autorité. 2) Bien qu'il prétende privilégier la pensée originale, le thomisme tente de la soumettre à une dictature intellectuelle. 3) Bien qu'il affirme démarquer la philosophie des contenus de la foi, le thomisme est tout entier soumis aux directives de cette instance qui a pouvoir de légiférer dans les matières concernant la foi, à savoir le SaintSiège. 4) Bien, enfin, qu'il se complaise à juger de haut la part d'irrationnel qui constitue le mystique et le religieux, le thomisme n'est qu'un avatar de l'autorité religieuse et il s'efforce, chaque fois qu'il dialogue avec la science, de prendre la part du sentiment, de l'ineffable et du mystère. La raison thomiste, au Québec, s'est prétendue démonstrative, originale, laïque et objective alors qu'elle s'est toujours révélée livresque, asservie, cléricale et passionnée. Ce trait selon lequel la pensée orthodoxe se révèle comme formation réac- 
tionnelle est structuré par un double mouvement qui nous semble très caractéristique des mécanismes de la pensée orthodoxe. Un premier geste, qui constitue la dénégation de toutes les appartenances extra-théoriques (religieuse ou politique) de la pensée orthodoxe est relayé par une revendication non moins opiniâtre de la scientificité. Il n'est peut-être pas de contexte où le discours réclame avec plus d'insistance qu'on lui confère les prédicats de la science que ces contextes où s'est instaurée une orthodoxie. Il faut souligner que la figure que le thomisme a tenté de prendre correspondait trait pour trait à des valeurs dont l'Église reconnaissait avec une acuité croissante qu'elles étaient partagées par une société d'où elle risquait d'être éventuellement expulsée. De là cet effort pour assumer, par formation réactionnelle, un visage qui correspondrait à des attentes pressenties. D'où aussi l'issue attendue de ce processus pathologique : le retour du refoulé et la névrose. On aurait tort de croire que ces névroses furent le lot d'associations marginales : tout le corps social en fut affecté et nous en souffrons encore: Comme le remarquait le Cardinal Villeneuve le troisième rôle de la philosophie, c'est :

Texte 10:

De subjuger les élites intellectuelles et par celles-ci toute la société dans les liens de la foi.

Mgr. J.-M.R. Villeneuve, Première session, p. 235.

\section{$2.4-$}

THÈSE NO 4 :

Une orthodoxie se définit par une volonté d'application des concepts. Son rapport avec la réalité sociale et historique est par principe immédiat.

Pour l'essentiel, toutes les contradictions que nous avons relevées précédemment peuvent se rattacher à l'antimonie liberté/servitude. Les tenants d'une orthodoxie doivent convaincre ceux à qui ils désirent l'imposer que malgré le caractère définitif de la doctrine proposée, un emploi est encore prévu pour le travail de la pensée craétrice et, en réalité, pour le travail de la pensée tout court. La solution à ce problème réside dans le concept d'application. Si le noyau de la doctrine est intangible, par le fait même que la doctrine a été instituée, reste à propager le message et surtout à appliquer la doctrine à la résolution de problèmes sociaux contemporains. La nouveauté ne viendra donc pas de la diversité des instruments mais de la multiplicité des applications d'un même 
outil à des problèmes différents. Les textes sont sur ce sujet formels et abondants. Nous pouvons à cet égard nous référer à ce texte déjà cité par lequel Mgr Pâquet expose le but des travaux qui se poursuivront dans l'Académie et où la part de l'application à des problèmes contemporains est considérable.

Il est impérieux que se rendre compte de la portée de cette quatrième thèse. Sa fonction est, pour l'essentiel, de contrer cette croyance naïve selon laquelle une orthodoxie, et de façon notoire le thomisme, se réalise sous la forme d'exercices et de travaux sans relation avec le contexte socio-politique. Nous n'avons pas l'intention de nier que bien des textes produits par le thomisme aient été abstraits; nous tenons cependant à soutenir avec force que le degré d'abstraction ou même d'impertinence de travaux qui sont soutenus par un volontarisme de l'application n'est jamais une mesure de leur influence réelle. Il faut à cet égard ne pas hésiter à prendre parti pour le paradoxe : bien loin de manquer la réalité sociale québécoise, ces travaux ont au contraire ajusté cette réalité à la mesure de leur inexistence. Littéralement pétrifiée par le spiritualisme de ses élites, c'est toute la société québécoise qui s'est mise à ressembler aux travaux qui se sont employés à la déréaliser.

Cette volonté d'application peut aussi nous servir à rendre compte de plusieurs autres traits du discours orthodoxe. Énonçons à cet égard le problème que doit résoudre l'application de ce discours. Ce problème a été maintes fois énoncé : comment utiliser pour résoudre les difficultés de l'époque contemporaine des solutions qui ont été produites à une époque antérieure à la nôtre? Le discours orthodoxe possède tout un arsenal de procédés pour parer à cette objection.

\section{Premier procédé}

Le premier de ces procédés est classique et bien connu de vous : il consiste dans une négation pure et simple du vecteur temps. Nous désignerons ce premier procédé par le terme d'acbronisation. Le texte le plus saisissant que l'on puisse citer sur ce sujet est celui du Père Simard qui rédige l'avant-propos du cinquième volume des Actes:

Texte 11:

À première vue, il peut sembler étrange aux hommes si agités de notre pays que quelques-uns 
d'entre eux se consacrent à la recherche et à la diffusion d'un enseignement donné au moyen-âge. Pourtant s'il est vrai que la déviation causée par la Renaissance et accrue par le Protestantisme, l'idéo. logie de 1789 et le mauvais emploi des sciences modernes, explique les pires de nos maux, il ne saurait être illogique de rétrograder jusqu'à l'époque où régnaient les doctrines les plus salutaires et les plus fécondes.

G. Simard, Cinquième session, p. III.

Un procédé d'achronisation peut prendre des variantes plus subtiles que la négation abrupte de l'histoire. Au lieu de nier le temps, on le redistribue autour d'un événement central, qui est naturellement la production du corpus orthodoxe (dans ce cas-ci, les Sommes de saint Thomas). L'œuvre de saint Thomas intègre tout ce qu'il y a de vrai dans les textes qui ont été écrits avant l'époque des Sommes et constitue l'origine de tout ce qui sera recevable dans les productions intellectuelles postérieures. Corrélativement, les penseurs qui précèdent saint Thomas peuvent être intégrés au camp thomiste, à titre de précurseurs, dans la mesure où leur discours peut être dit vrai. Quant à ceux qui suivent saint Thomas, leur prétention à la vérité est exactement mesurée par leur inféodation au camp thomiste. Cette redistribution du temps donne lieu à des travaux dont le caractère loufoque peut être évalué à l'embarras des éloges qu'ils suscitent :

\title{
Texte 12:
}

\begin{abstract}
Avec le substantiel plat métaphysique qu'on doit au chanoine Président de notre Académie, nous avons eu ce soir ce que joserais appeler un dessert quelque peu fantaisiste de pré-thomisme. En effet les deux saints dont on nous a raconté la querelle - il s'agit de Saint Augustin et de Saint Jérôme - ne furent thomistes que bien avant la lettre et asse\% peu quant à l'esprit.'

Mgr J.-M.-R. Villeneuve, Dixième session, p. 185.
\end{abstract}

Ce pauvre Cardinal commençait à s'y connaitre ; il avait dû. deux années auparavant, faire l'éloge d'un jeune clerc qui avait vu dans Athanase, le patriarche d'Alexandrie, un autre des précurseurs de saint Thomas.

5. Mgr Villeneuve avait, à cette époque, été fait cardinal. 
Texte 13: $\quad$ Et voilà comment, ce soir, tout en nous parlant d'un docteur de l'Église qui précéda presque d'un millénaire l'Angélique Docteur, le R.P. Conférencier a prétendu faire du thomisme avant la lettre, et appeler saint Athanase le saint Thomas de son siècle. $\mathrm{Si}$, en effet, le grand patriarche d'Alexandrie commença par secouer les théories antérieures ... il aura été un thomiste précurseur...

Mgr J.-M.-R. Villeneuve, Neuvième session, p. 51-52.

Les deux exemples que nous venons de donner sont relatifs à des penseurs qui ont précédé saint Thomas. Pour ce qui est des efforts pour rattacher à saint Thomas des réalisations qui lui sont considérablement postérieures, la palme doit être décernée à $\mathrm{Mgr}$ Lebon qui, en 1934, année où l'on discutait beaucoup des bienfaits de la Société des Nations, présenta un texte à la session annuelle de l'Académie, où il tenta de montrer que les doctrines contemporaines sur la paix internationale dérivaient en droite ligne de la doctrine de saint Thomas par l'intermédiaire de Suarez, Vittoria et Taparelli. Le triomphalisme naif du commentaire que fit Mgr Lamarche du texte de $\mathrm{Mgr}$ Lebon nous amènerait aujourd'hui à sourire :

Texte 14:

Aujourd'hui, l'Église par ses universités est encore le meilleur facteur de la paix internationale. Le jésuite Taparelli fut le précurseur de la Société des Nations. Cette doctrine des Universités est étendue au monde entier par les documents pontificaux.

Mgr C. Lamarche, Cinquième session, p. 241.

\section{Second procédé}

Le premier processus que nous avons décrit constitue d'une certaine façon un processus d'abstraction: le discours de l'orthodoxie est systématiquement abstrait de tout contexte historique - que ce soit son contexte d'origine ou son contexte d'application - dont la description serait suffisamment précise pour le faire paraître anachronique et lui retirer sa pertinence. À ce premier processus d'abstraction qui porte sur le dehors du discours orthodoxe - les circonstances de son origine ou celles de son application s'ajoute un second procédé d'abstraction qui, cette fois, porte sur ce 
discours lui-même. Nous nommerons ce second procédé, dont l'importance est encore plus considérable que le premier, l'extraction de la doctrine.

La croyance selon laquelle le rapport entre une orthodoxie et la pensée du maître dont elle se réclame est immédiat et transparent ne résiste jamais à l'examen de la production réputée orthodoxe. Un indice de la distance qui sépare la pensée d'un maître et la doctrine orthodoxe qui est extraite de l'ensemble de ses textes peut être vu dans ce titre que le Père Simard donne à l'une des sections de sa communication sur les "thomistes et saint Augustin". Le titre de cette section est "Les caractéristiques de l'augustinisme de saint Augustin ". (Simard, p. 26, Sixième session). Ce texte sug. gère que, en dépit des apparences, saint Augustin lui-même n'occupe pas une position privilégiée au sein de l'augustinisme : l'augustinisme constitue une doctrine qui transcende non seulement les conditions historiques de sa production mais aussi sa base textuelle originaire et à laquelle divers auteurs sont comparés selon les modalisations propres qu'ils lui font subir. L'augustinisme de saint Augustin pourra ainsi être contrasté avec celui de saint Irénée ou de saint Thomas. Or, ce qui vaut de l'augustinisme vaut évidemment aussi du thomisme. Nous dirons que de façon générale le rapport d'une doctrine instituée avec le penseur qui lui a donné naissance est d'ordre essentiellement sélectif. Nous avons donné le nom d'extraction de la doctrine à cette sélection, dans la pensée d'un maître, de ce qui doit devenir doctrine instituée.

Ce processus de sélection obéit, pour l'essentiel, à une règle d'abstraction. Le projet d'achronisation antérieurement décrit et qui avait pour fin d'estomper la différence entre les divers contextes socio-culturels se prolonge d'une tentative corrélative pour éterniser la doctrine, en rejetant, d'une part, toutes les propositions qu'elle contient qui seraient susceptibles d'être datées dans la chronologie de l'évolution des idées et en accroissant, d'autre part, si cela est possible, le degré de généralisation de propositions déjà sélectionnées en vertu de leur caractère abstrait. Ces deux variantes de ce que nous désignerons dorénavant comme un processus d'éternisation et qui accomplit au niveau du contenu d'une doctrine ce que le procédé d'achronisation accomplit au niveau de son contexte d'origine opèrent de la façon suivante. 
La première variante du procédé d'éternisation accomplit une opération qui est avant tout négative. Usant d'une distinction, introduite par Maritain et reprise par le cardinal Villeneuve l'enrobement d'une pensée et son corps véritable, on rejettera du côté de l'enrobement, qui, comme son nom l'indique, ne concerne que les propositions non essentielles, tous les énoncés auxquels il est possible d'asigner une date précise. Ces énoncés appartiennent en général à la classe des énoncés qui sont tributaires des connaissances scientifiques d'une époque et dont on peut dire que les positions qu'ils expriment sont dépassées. Cette classe d'énoncés a cependant une plus large extension que celle des énoncés à caractère scientifique. De façon générale la distinction entre le corps propre d'une doctrine et son revêtement cautionne le rejet hors du noyau essentiel de la doctrine de toutes les propositions formulées par le maitre qui ne sont pas conformes aux fins qui sont poursuivies par l'institution de sa pensée. Le résidu de cette première opération d'épuration est censé constituer la part éternelle de la pensée du maître et c'est elle qui est instituée comme doctrine contraignante. Le caractère déterminant des énoncés qui constituent la doctrine ainsi éternisée est leur extrême généralité. Si, comme nous l'avons déjà dit, cette généralité n'est pas jugée suffisante on s'emploiera à l'accroître encore. Un texte du cardinal Villeneuve est à cet égard particulièrement révélateur.

Texte 15:

"Deux quantités égales à une troisième sont égales entre elles " est un principe mathématique évident par lui-même. Toutefois il se résout en ce principe métaphysique dont il n'est qu'une sorte de contradion (sic) "Deux êtres identiques à un même troisième sont identiques entre eux $\%$. Les principes des sciences ne sont en définitive que des applications morcelées et des incarnations restrictives de l'être universel et des évidences transcendantales ".? Mgr J.-M.-R. Villeneuve, Première session, p. $223-24$.

Les commentaires que l'on peut faire à propos de ce texte où l'on pense toucher du doigt le néant sont très nombreux. Je les

6. "Il ne s'agit pas de s'attarder à retenir les éléments matériels et caducs de la synthèse thomiste, par exemple, la physique expérimentale dans laquelle les anciens, selon le mot de Maritain, enrobaient (souligné dans le texte), faute de mieux, leur métaphysique. "Villeneuve, Première session, p. 246.

7. "Contradion" est sans doute une coquille. Il faudrait lire "contraction". 
ferai à l'occasion de l'énoncé d'une cinquième thèse sur la nature d'une orthodoxie.

THÈSE NO 5: La généralité des contenus doctrinaux d'une orthodoxie lui confère une plasticité telle qu'elle peut être utilisée pour légitimer ou pour condamner n'importe quelle prise de position.

Cette thèse constitue le commentaire annoncé du texte précédent. Il ne suffit pas de dire que la pensée qui s'exprime dans ce texte est grossièrement verbale ; il faut rendre compte des effets de ce type de pratique intellectuelle. Dans des textes de ce genre, - et ils sont nombreux, - les énoncés manquent tellement de spécificité que la pensée et le discours semblent y tendre vers leur propre abolition. Au sens le plus fort du terme, on parle le langage d'une orthodoxie pour ne rien dire. La conséquence de ce truisme est meurtrière : si les contenus doctrinaux d'une orthodoxie sont en réalité vides, il n'est absolument rien à quoi ils ne sauraient être adaptés et, en conséquence, il n'est pas de limite à son utilisation pour le pouvoir qui l'a mise en place. Je donnerai maintenant trois exemples pour illustrer le degré de plasticité qu'on a ainsi conféré aux contenus du thomisme. Lorsque j'ai affirmé que ceux-ci pouvaient être utilisés pour légitimer n'importe quoi, on verra que je n’ai rien exagéré.

\section{Premier exemple}

Mon premier exemple constitue moins en lui-même un exemple isolé qu'il n'a valeur de symbole du procédé le plus courant qui opère dans ces textes et que je désignerai comme l'emploi de citations fétiches. Une citation fétiche est un renvoi à un texte de saint Thomas, - rarement puisé dans l'œuvre de saint Thomas lui-même, on l'a plutôt trouvé déjà cité par un commentateur, - - qui n'est opéré que pour la forme et par déférence aux prescriptions de l'autorité religieuse. L'écart de généralité entre les propositions de ce texte de saint Thomas et la thèse défendue par l'auteur qui le cite est tel qu'il est littéralement impossible de vérifier si le texte cité est favorable ou non à la thèse proposée par l'auteur. 
Démontrons cela. Soit ce texte, dont l'auteur énonce dans son introduction les deux prémisses et la conclusion à laquelle il parviendra, ayant établi ces prémisses. Voici les prémisses. Je révélerai la conclusion plus tard.

Texte 16:

Il y a lieu, d'abord, de bien envisager un fait, le fait des professions. Il faut ensuite situer à leur rang les professions dans l'organisation de la vie sociale.

L. Pelland, Septième session, p. 145-46.

La première prémisse est indiscutable : les professions constituent un fait social établi. Tout va donc se jouer sur la seconde prémisse. On va recourir à saint Thomas pour éclairer ce problème d'ordonner les diverses professions.

Texte 17:

Dans la première partie de sa Somme Tbéologique, saint Thomas d'Aquin, après avoir défini et analysé l'homme au regard de Dieu et en face de la création, constate que tout l'univers créé est en quelque sorte au service de l'humanité, appelé à une fin surnaturelle et qui doit être mise à même d'atteindre sa fin suprême par une série de fins intermédiaires. Tout le problème de la vocation temporelle et de l'orientation professionnelle est là.

L. Pelland, Septième session, p. 149.

De ce texte de saint Thomas l'auteur tire que " les professions existent parce que les hommes sont divers. .. ". "De là, poursuit notre auteur, l'inégalité des conditions et l'extrême diversité des professions" (p. 149-50). Saint Thomas nous a donc appris que la diversité des professions correspondait à la diversité des aptitudes. Il est temps de conclure. Quelle est, je vous le demande, la conclusion qui doit être tirée de notre raisonnement et du texte de saint Thomas? Cette conclusion, que personne sans doute n'aurait trouvée, la voici :

Texte 18:

Ce point réglé, la conclusion s'impose : la médecine et les professions en général ne peuvent pas être étatisées. $^{8}$

L. Pelland, Septième session p. 146

8. L'auteur avait déjà énoncé sa conclusion avant d'entreprendre d'en démontrer les prémisses. C'est pourquoi la conclusion que nous citons apparaît sur une page qui précède celle où apparâit son argument en faveur de cette conclusion. 


\section{Deuxième exemple}

Un voyage au pays de l'Académie canadienne Saint-Thomas ne constitue pas en général un itinéraire plaisant. Il est cependant des textes où l'équanimité du chercheur doit faire place au dégoût. À la session de 1936, le R.P. Arthur Caron, vice-recteur de la Faculté de Droit canonique de l'Université d'Ottawa, présente une communication sur « Les droits de la civilisation et l'occupation des terres non civilisées ". Ce sujet, en apparence abstrait, est d'une brûlante actualité : l'année précédente, l'Italie de Mussolini a envahi l'Éthiopie et le Duce a enfin connu la volupté de la gloire militaire. Les canons italiens ont eu raison, bien qu'avec difficulté, des flèches des habitants de l'Abyssinie. La navrante astuce du Père Caron consiste à renverser la question du droit des peuples civilisés à l'occupation des terres non civilisées en celle du droit des peuples soi-disant non civilisés à l'occupation par un peuple qui leur apportera les bienfaits de la civilisation:

Texte 19:

Ces préliminaires posés, examinons le problème du premier point de vue, savoir : les droits des peuples incultes à la civilisation.

A. Caron, Septième session, p. 47.

Or, le Père Caron n'bésitera pas à modifier la doctrine de certains commentateurs de saint Thomas sur ce sujet, en particulier de Taparelli, a fin de défendre avec un entêtement qui lui fait honneur le droit fondamental des nègres à être conquis par les blancs et à participer ainsi aux bienfaits de la civilisation. L'ignominie atteindra son point culminant dans ce texte où il se révélera que les peuples riches mais incultes n'ont pas seulement le droit à la civilisation mais qu'ils ont en stricte charité chrétienne le devoir de partager leurs richesses avec les peuples plus civilisés mais qui ont besoin de Lebensraum.

Texte 20:

L'autorité ethnarchique, en vertu d'une justice distributive suréminente qui doit présider à l'équitable partage de la propriété humaine entre les nations, aurait le droit et le devoir de faciliter l'expansion d'une nation surpeuplée et incapable de nourrir tous ses fils en lui ouvrant des terres nouvelles dans les régions inexploitées de l'univers. Cajetan ne soutient-il pas que même dehors des cas d'extrême nécessité, l'autorité sociale aurait le droit de distri. 
buer aux pauvres le superflu dont les riches ne veulent pas se départir? (39). Il suffit de transposer ce principe sur le plan international, et nous ne voyons pas ce qui le rendrait inopérant.

D'autre part, le bien commun international et la justice sociale internationale ne font-ils pas aux nations un devoir de consentir au partage de leur superflu et de leur surabondance entre les membres les plus indigents de la communauté des peuples? (39). "Sed in primo casu, cum quis habet de superfluo ... " cf. Commentarium in IIam IIx, qu. 118 , A, 4, n. 3 .

A. Caron, Septième session, pp. 57-58.

Aussi incroyable que cela puisse paraître, il faut préciser que dans ce texte de Caron les pays riches qui doivent partager leurs richesses seraient des pays comme l'Abyssinie, c'est-à-dire l'Éthiopie d'aujourd'hui, qui est, comme on sait, une terre de famine. Fait alors figure de pays pauvre l'Italie fasciste, qui était cependant assez riche pour envoyer un corps expéditionnaire de cinquante mille hommes en Espagne, afin d'aider Franco à gagner sa guerre civile. L'argument de Caron aurait pu être utilisé en 1939 pour légitimer l'invasion de la Pologne (pays riche) par l'Allemagne (pays pauvre) et, de façon plus générale, pour faire l'apologie de tous les impérialismes. Tout cela, comme le montre la note en bas de page (note 39) que nous avons commencé à citer, s'autorise d'une citation de Cajetan, commentateur latin de saint Thomas.

\section{Troisième exemple}

Le troisième de nos exemples est tiré d'un texte de l'abbé Alphonse-Marie Parent, - celui là même qui devait donner son nom à un célèbre Rapport sur l'éducation, - qui a pour titre "Autour du racisme". Cette communication de l'abbé Parent s'inscrit dans le contexte suivant: en 1938, dans une lettre adressée à tous les recteurs des universités catholiques, la Sacrée Congrégation des Études, Séminaires et Universités, qui relève du Saint-Siège, déclare insoutenables huit des propositions soutenues par le racisme. La troisième de cés propositions, la plus fondamentale peut-être, s'énonce comme suit :

C'est du sang, siège des caractéristiques de la race, que dérivent, comme de leur source principale, toutes les qualités intellectuelles et morales de l'homme. 
Le texte de Mgr Parent va consister à montrer qu'en dépit de la prescription du Saint-Siège, cette proposition comporte une large part de vérité.

Texte 21:

Il est faux de prétendre qu'il n'existe aucun rapport entre le sang et les qualités intellectuelles et morales d'un individu ou d'un peuple...

Il est également faux et par trop simpliste de déclarer le racisme absurde sous prétexte que tous les hommes et tous les peuples sont égaux par nature...

Or nier les rapports entre le sang et les qualités intellectuelles et morales de l'homme, ou les passer sous silence, implique une conception de l'homme qui ne cadre guère avec celle d'Aristote et de saint Thomas. A-M. Parent, Dixième session, p. 99.

L'affaire étant assez chaude, l'abbé Parent va joindre à l'autorité de saint Thomas et d'Aristote celles de saint Albert le Grand et de Cajetan pour parvenir à la conclusion suivante, qui suit une citation de plus de deux pages de saint Thomas:

Texte 22:

Il n'est pas étonnant alors que la proposition condamnée par le Saint-Siège s'exprime ainsi : ex sanguine, veluti et potissimo fonte... Il n'en est pas moins vrai que le sang, le corps et plus généralement la nature est cause partielle, "secumdum inchoationem", des dispositions spécifiques et individuelles, intellectuelles et morales des individus aussi bien que des nations telles que définies plus haut.

A.-M. Parent, Dixième session, p. 106.

En gros, ce que condamne l'Église lorsqu'elle dénonce la troisième proposition du racisme, c'est la croyance que le sang constitue la source ultime ou principale des qualités morales et intellectuelles d'une nation ou d'un peuple. On ne saurait cependant arguer de la position de l'Église, c'est là le point majeur du texte de l'abbé Parent, pour prétendre qu'il n'y a aucun lien entre le sang et la qualité d'un homme. Ce lien est en réalité si immédiat que dans le paragraphe qui suit immédiatement la conclusion que nous venons de citer, l'abbé Parent pourra écrire que : 
Texte 23: $\quad$ Et de même que certains individus sont plus aptes par nature à se gouverner et à gouverner les autres, de même certaines nations sont plus aptes par nature à se gouverner et à gouverner les autres.

A.M. Parent, Dixième session, p. 106.

Ce dernier passage, faut-il le souligner, est l'expression canonique - par excellence - de la position raciste.

Mais pourquoi cette prise de position, de la part d'un abbé, en faveur du racisme? La raison en est pourtant simple. Cette insistance sur l'inégalité naturelle entre les hommes a pour résultat d'accentuer la nécessité d'une intervention du surnaturel dans les affaires humaines et de lui réserver le privilège de polariser tous les efforts des hommes pour réaliser le règne de la justice sur la terre. Dit en clair, dans un monde où l'inégalité consitute la loi, seule une Église qui transcende l'ordre naturel peut légitimement déployer la bannière de l'amour universel. Ainsi se trouvent disqualifiées des idéologies comme celles que représente le communisme et qui prétendent se fonder sur la fraternité naturelle de tous les hommes. Cette prétendue fraternité n'est en fait qu'une perversion de la nature qu'engendre la haine:

Texte 24:

Elles (les distinctions précédemment faites) nous montrent en même temps la profanation que commettent les égalitaristes qui convertissent la nature en pure apparence, voire qui nient la nature, lui substituant un pervertissement du surnaturel, la fraternité universelle laïciste. Or l'égalité et la fraternité qui n'ont pas pour principe le Christ-Roi et la Reine des Anges ne pourront jamais être que des contrefaçons issues de la haine, de l'envie, de la rancune.

A.-M. Parent, Dixième session, p. 122.

Le sentiment auquel donne voix le texte de l'abbé Parent, aussi inhabituel qu'il nous paraisse, est loin d'être marginal parmi les membres de l'Académie thomiste canadienne. Le ton du passage du texte de l'abbé Parent que nous avons cité apparaît même mesuré si nous le comparons à l'âpreté des propos que tenaient ces premiers professeurs laïques que l'autorité religieuse se résolut à introduire dans l'institution philosophique canadienne- 
française. À témoin ce texte de Charles de Koninck, où il s'efforce d'assimiler l'un à l'autre le personnalisme et le marxisme en citant des paroles de Jacques de Monléon.

Texte 25:

Comme nous disait Jacques de Monléon: "Remarque que les prétendus personnalistes qui mettent la personne au-dessus du bien commun ne peuvent plus voir dans celui-ci le lien des personnes. Dès lors, ils remplacent ce lien par un autre, par une sordide fraternité (nous soulignons) qui unirait immédia. tement les personnes entre elles: comme si chaque personne était un bien commun pour toutes les autres. Ce qui revient à faire de chaque citoyen un tyran, amans seipsum magis quam civitatem." Tel fut pourtant l'idéal de Marx.

C. de Koninck, Dixième session, p. 102.

La mesure de l'information apportée par un texte pourrait s'évaluer, selon plusieurs théoriciens contemporains, par l'improbabilité des relations que ce texte établit entre certains de ses items lexicaux. Si tel était le cas, la conjonction, assez inattendue il faut le reconnaître, de l'épithète "sordide " et du substantif " fraternité " devrait retenir notre attention et pourrait même constituer un test de validité (parmi d'autres) pour la description de ce que fut l'aristotélico-thomisme dans notre société. Cette description pourrait en effet se donner pour l'une de ses fins, celle de découvrir l'ensemble des conditions tant discursives qu'extra-discursives d'émergence et d'acceptabilité d'un tel énoncé.

$2.6-$

THÈSE NO 6: $\quad$ Ce que la pensée orthodoxe est appelée à légitimer et à défendre ne dépend pas de ses contenus doctrinaux mais est déterminé par les intérêts de l'instance qui l'a instituée.

A la lumière de ce qui précède, cette thèse devrait paraître évidente. Il faut néanmoins l'énoncer pour avoir l'occasion de répondre à une objection, qui, à ce point de l'exposé, pourrait être faite. Cette objection serait la suivante. La thèse 5 pose que les contenus d'une orthodoxie sont suffisamment plastiques pour être 
adaptés à n'importe quelle fin. Il s'ensuivrait que le pouvoir de contrainte que confère l'institution d'une pensée doit être reconnu comme nul : si cette pensée est vidée de tout contenu spécifique, elle ne saurait contraindre personne à adopter tel point de vue plutôt que tel autre. Or il faut répondre à cette objection que la plasticité des contenus d'une orthodoxie décuple au contraire le pouvoir de contrainte qui peut-être retiré de l'institution d'une doctrine. Le vide des contenus a tôt fait d'être relayé par le plein de l'intérêt. Précisons, en effet, que la mise en place d'une orthodoxie repose non pas sur un seul geste institutionnel mais en réalité sur deux. Un premier geste consiste dans l'institution d'une doctrine. C'est ce premier geste, qui a fait l'objet de notre thèse initiale, qu'il nous faut maintenant compléter par la description d'une seconde opération. L'instauration d'une orthodoxie redouble l'institution d'une doctrine par la détermination de ceux qui ont le pouvoir de l'interpréter et par l'officialisation de ce pouvoir. Or, ce pouvoir d'interprétation coöncide dans le cas d'une orthodoxie avec un pouvoir infini de modification et d'assignation de contenu. Ce que nous tentons de dire ici tombe sous le sens : si les énoncés d'une doctrine sont d'une généralité et d'une plasticité telles qu'on peut les déclarer à toutes fins pratiques vides de contenu, les interpréter consiste effectivement à leur connférer ce contenu qui leur manque. On pourrait encore une fois formuler sous la forme d'une dernière thèse le résultat auquel nous venons de parvenir :

THĖSE NO 7: Le pouvoir d'interpréter le texte de l'orthodoxie coïncide avec celui de l'écrire et en conséquence, de déterminer son contenu.

Dans le contexte d'une orthodoxie, l'interprétation et l'écriture sont des opérations parfaitement réciproquables. La mise en place d'une orthodoxie n'instaure jamais la domination d'un maître mais celle de ses exégètes. Détenant le pouvoir d'interpréter la doctrine, ce sont aussi ces exégètes qui s'arrogent le pouvoir de décréter qui lui est fidèle et qui ne l'est pas. Or puisque leur pouvoir d'interpréter une doctrine se confond avec leur pouvoir de la réécrire, il n'est absolument aucune limite à l'arbitraire du jugement qu'ils peuvent porter sur le degré de conformité d'une pensée quelconque à la doctrine dont ils fixent, au gré des intérêts qu'ils défendent, le contenu. La doctrine orthodoxe est donc minima- 
lement contraignante pour ceux qui ont le pouvoir de l'interpréter et maximalement contraignante pour ceux qui sont soumis à ce pouvoir. Alors qu'il était légitime pour un conférencier de l'Académie canadienne Saint-Thomas d'Aquin de prendre parti pour le racisme, il n'était même pas permis aux lä̈ques de se reconnaître comme des frères.

3 - Nous aimerions maintenant, dans la dernière partie de cet exposé, dire quels sont les effets de la mise en place d'une orthodoxie. Nous le ferons en deux temps, présentant d'abord quelques effets généraux et terminant par la discussion de quelques effets particuliers à la société québécoise.

3.1 - L'effet le plus général, et celui dont découlent tous les autres, de l'instauration d'une orthodoxie consiste dans une déperdition du langage. Qu'on y prenne garde cependant, nous ne faisons pas ici référence à une banale réduction au silence d'un groupe de personnes au profit d'un autre. Nous voulons plutôt signifier que l'expression linguistique elle-même dégénère et perd sa nature de signe. Le propre d'un signe linguistique est en effet d'être porteur d'un sens. Cette liaison d'un signe avec son sens est habituellement contraignante pour la masse des sujets parlants. Lorsque, par exemple, nous apprenons une langue étrangère, nous ne pouvons pas à notre gré faire correspondre aux divers termes du vocabulaire de cette langue n'importe quel mot français comme étant son sens. Au contraire, cette mise en correspondance constitue essentiellement un phénomène réglé auquel il convient de se soumettre si nous voulons parler une langue étrangère. Le propre de l'établissement d'une orthodoxie est de rompre cette liaison des signes avec leur sens reconnu et de substituer à la contrainte sémantique commune des signes linguistiques l'opération d'une volonté particulière, déterminée par l'intérêt du groupe ou de l'instance où elle inhère. Cette rupture de la liaison entre les signes et le sens qu'ils expriment habituellement fait régresser le langage au statut de simple chose - un bruit en mal de sens - et au lieu d'exprimer des concepts le langage devient essentiellement l'instrument, au sens fort de ce terme, d'une volonté. Cette volonté étant particulière et son opération arbitraire, les liaisons qu'elle se décidera, de façon essentiellement provisoire et opportune, à établir entre certains signes et certains contenus de signification deviennent imprévisibles. Ce qui est le comble de l'orthodoxie aujourd'hui deviendra blasphème demain, au gré de la décision du 
plus puissant de ses exégètes. Une pratique meurtrière du soupçon, qui se nourrit de l'obsession d'être tenu responsable de son discours, détermine une perte du langage qui, on ne l'a pas assez remarqué, affecte autant ceux qui détiennent le pouvoir de parler que ceux qui leur sont soumis. Cette perte du langage se manifeste de trois façons selon le rang qu'occupe dans la hiérarchie des pouvoirs celui qu'elle affecte.

Première façon : ceux qui sont complètement exclus de la structure des pouvoirs décident habituellement de se taire ou plutôt de n'émettre aucun son.

Seconde façon: ceux qui se risquent à parler, même s'ils sont liés avec le pouvoir, ne le font plus que par personne interposée. C'est la pratique du renvoi indéfini à la chaîne des commentateurs, qui nous frappe tellement lorsque l'on aborde par exemple la description des textes produits par l'orthodoxie thomiste. Un thomiste cite Maritain, qui s'appuie sur Cajetan, exposant lui-même un commentaire que fait saint Thomas d'une thèse d'Aristote. Personne ne parle dans ce processus, soulignons-le avec force, car tout le monde cite. Cette pratique obsessive de la citation qui caractérise le texte de l'orthodoxie n'est nullement adventice. Elle réalise au contraire le rêve du penseur orthodoxe : réussir à contraindre tout en parlant pour ne rien dire, afin de n'être responsable de rien. Je ne dis rien lorsque je cite, c'est l'autre qui parle à ma place. ${ }^{\prime}$

Troisième façon : c'est celle que se réserve l'élite du pouvoir. Le discours devient un mode de l'agir :

9. Il y a évidemment lieu de distinguer entre deux usages profondément différents de la citation. Un texte peut être allégué de façon documentaire pour valider ce qui en est affirmé au niveau d'un métadiscours. Toutes les citations qui apparaissent dans le cadre de cet exposé sont invoquées au seul titre de documents. Le trait propre de l'usage documentaire des textes est de mettre en suspens leur valeur de vérité. Il est aussi possible d'utiliser de facon autoritaire une citation : celle-ci est alors concue comme l'expression d'une vérité découverte par un maitre et le discours qu'elle a pour fonction de valider ou d'appuyer n'est plus un métadiscours mais un discours de premier degré. Dans l'usage autoritaire, la citation n'apparaît pas pour fonder ce qu'un discours affirme d'un autre discours; elle intervient pour montrer que ce qu'un discours affirme $d u$ monde est véridique parce que conforme au corpus des vérités reconnues sur le monde, à savoir la pensée d'un maître. C'est de cette façon autoritaire que la citation est, la plupart du temps, utilisée dans les Actes de l'Académie canadienne Saint-Thomas d'Aquin. 
Texte 26 :

Je veux donc exposer maintenant comment non seulement l'universitaire-philosophe, de ses hauteurs, doit pouvoir juger les sciences et les contrôler, mais d'une façon prochaine les stimuler et les diriger. Mgr J.-M.-R. Villeneuve, Première session, p. 221.

Texte 27:

Le troisième rôle qui lui revient (la philosophie) dans nos universités chrétiennes, c'est, je l'ai dit, après avoir créé l'esprit universaliste et formé le spécialiste d'envergure, de subjuger les élites intellectueelles, et par celles-ci toute la société, dans les liens de la foi.

Mgr J.-M.R. Villeneuve, Première session, p. 235.

(déjà cité, TEXTE 10, p. 14).

Le penseur orthodoxe a cessé de parler. Il a mieux à faire : il gouverne, subjuge, met en ordre ou trace la ligne juste, bref il agit.

3.2- Le texte précédent laisse entendre que l'Académie thomiste était tout autre chose, à tout le moins au niveau de l'énoncé de ses fins, qu'une société désintéressée composée de prélats ou de professeurs érudits qui se tenaient le plus éloignés possible des distractions de ce monde. Le conditionnement idéologique qui s'est élaboré au sein de cette société a-t-il été efficace ? Cela ne fait pour moi aucun doute. Un indice de cette efficacité : le texte qui, je crois, est le plus souvent cité dans les Actes de l'Académie, est cette phrase fameuse de Maritain selon laquelle le monde a plus besoin de métaphysique que de charbon. Or cela, il n'est pas exagéré de dire que la société québécoise a fini par le croire puisqu'elle s'est bien davantage employée à entretenir l'espérance métaphysique qu'à construire des hauts fourneaux et quelle affirme encore aujourd'hui privilégier la souveraineté culturelle sur l'indépendance économique. Pour l'essentiel, l'installation au Québec d'une pensée reconnue orthodoxe nous a fait acquérir certains habitus, qu'il me reste à énumérer brièvement. Cette énumération n'est évidemment pas exhaustive.

3.21 - Qu'avons-nous donc appris dans cette Académie canadienne Saint Thomas? Nous y prîmes d'abord l'habitude de la modestie. 
Il serait bien téméraire d'espérer faire avancer une question qui exerce la sagacité des plus grands esprits... Contentons-nous d'espérer à ne pas trop l'embrouiller et restons le plus près possible de la pensée du Maître...

F. Yelle, Troisième session, p. 226.

Et notre tâche est terminée. Nous ne prétendons pas le moins du monde avoir apporté des lumières nou. velles à la solution du difficile problème de la connaissance. Telle n'était d'ailleurs pas notre inten. tion. Modeste vulgarisateur, nous avons voulu mettre en évidence quelques points fondamentaux de la théorie thomiste concernant le fonctionnement des espèces intelligibles.

L.-P. Fafard, Quatrième session, p. 109-110.

Nous n'avons pas la prétention de suivre ces philoso- phes dans l'exposition de leur système. Cela nous entrainerait trop loin : nous voulons tout simplement revenir à l'idéal chrétien de la "bonne vieille morale " (sic) qui apporte la solution aux problèmes de tous les temps.

P. Périer, Quatrième session, p. 230.

$\mathrm{J}$ 'ai voulu répondre à cette question et à divers points subsidiaires qu'elle soulève en consultant les meilleurs autorités : chez le modeste vulgarisateur que je prétends être la bouche parle de l'abondance du livre.

M.A. Lamarche, Cinquième session, p. 29-30.

Notre intention n'est pas d'embrasser cette question dans toute son ampleur : elle déborderait le cadre de ce modeste travail ... Nous voulons étudier seulement la nature de la perfection chrétienne d'après les principes de saint Thomas d'Aquin.

A. Desnoyers, Cinquième session, p. 142.

Loin de nous la prétention de bâtir une théologie de l'action catholique: notre témérité n'atteint pas ce niveau. Notre ambition, beaucoup plus modeste, se limite à un travail d'approche et à quelques défrichements.

A. Ferland, Septième session, p. 114. 
Nous n'avons ni la prétention ni l'espoir d'apporter, aux difficultés ardues où s'agitent les nations, une réponse nouvelle et libératice. Nos visées sont beaucoup plus modestes. Ce sont celles du disciple qui n'a pas d'autre ambition que d'extraire des ouvres de son maître saint Thomas d'Aquin quelques principes propres à éclairer...

A. Caron, Septième session, p. 25-26.

le rôle qu'on m'a attribué ..., c'est de poser la question plutôt que de la résoudre...

A. Sylvestre, Douzième session, p. 109-110.

J'interromps cette énumération qui pourrait être indéfiniment poursuivie. Cette fureur à ne pas être est sans exemple. Comme on le voit, la propension de notre communauté pour l'humilité n'est pas le fruit du hasard. Nous avons, comme on dit, de qui tenir.

3.22 -- Cette passion pour l'impuissance devait évidemment influer sur la conception du travail et de la production intellectuels que se faisait à cette époque l'Université québécoise. Une habitude que nous acquîmes, en second lieu, à ces sessions de l'Académie thomiste, fut de transformer en un rite ou en un cérémonial la communication des résultats de la recherche philosophique ou, plus simplement, théorique. Or le trait particulier au langage rituel est la dissociation qu'il opère entre le contenu de ses formules et sa valeur d'acte ou, encore, sa fonction. Les formules, pour prendre un exemple simple, par lesquelles s'inaugure une session du parlement canadien constituent un geste de déférence à l'égard de la Couronne britannique mais ne recèlent que peu de sens véritable pour les Canadiens (à l'exception peut-être de quelques loyalistes, s'il en est encore). D'une façon analogue, il semble que les textes produits par l'Académie thomiste se soient investis tout entiers dans leur fonction d'allégeance à l'autorité ecclésiastique, au détriment de leur contenu théorique jugé, sauf sous le chapitre de sa conformité, sans grande importance. La production théorique a donc été de façon croissante dissociée de la tâche de dire le vrai et de faire progresser la recherche. Strictement conçue sous l'espèce des servitudes inévitables liées à la pratique d'une profession et à l'occupation d'une place dans la hiérarchie sociale, la communication philosophique n'a bientôt plus constitué qu'un cérémonial dont le seul but était la manifestation formelle d'une capacité à remplir un poste et d'un désir de s'y maintenir. Un bon exemple de 
cette ritualisation est donné par la communication de Châl les de Koninck intitulée "Marie et la sagesse" (Onzième session, p. 218-247).

Quelle qu'ait été la fin de ces rituels, ceux qui s'en faisaient les officiants recevaient leur juste salaire dans la gratitude de l'autorité ecclésiastique. On peut à cet égard citer le compliment qu'adresse a Charles de Koninck le Provincial des Dominicains, qui présidait la séance au cours de laquelle fut présenté "Marie et la sagesse ".

Texte 29:

J'ai également l'honneur et le plaisir de vous représenter tous, en ce moment, Mesdames et Messieurs, pour exprimer nos remerciements à $\mathrm{M}$. le Professeur de Koninck, le conférencier de ce soir.

M. le Professeur de Koninck nous a à la fois charmés, instruits et édifiés par son docte travail : "Marie et la Sagesse".

M. de Koninck est maître en sagesse naturelle, et, nous l'avons constaté, il se meut avec aisance dans la Sagesse surnaturelle.

Si nous étions encore au temps des luttes entre le Sacerdoce et l'empire, au sujet de leurs empiétements réciproques, l'empire lui-même serait surpris et le sacerdoce scandalisé, en voyant pareille intrusion d'un laï dans le domaine de la Mariologie. D'autant que dans le cas de $M$. de Koninck, il s'agit d'un empiétement, non seulement en superficie mais en profondeur. Et n'est-ce pas précisément cette avidité d'atteindre aux conclusions extrêmes des principes naturels et du donné révélé qui étonne, j’allais dire qui détonne de la part d'un savant que beaucoup, du moins en dehors de Laval, croyaient confiné dans le pur Aristotélisme?

Toutefois, après l'avoir entendu exposer avec cette force déductive et cette ampleur, traversés d'un souffle de piété, les grandeurs de la Vierge et les miséricordes de Dieu à son égard, si la surprise demeure, le scandale disparaît.

Il disparaît pour faire place à la reconnaissance. $M$. le Conférencier, comme le disait l'autre soir l'Hono- 
rable Juge Thibaudeau Rinfret à l'un de vos collègues, des hommes tels que vous, on ne les loue pas, on les remercie.

Pie-M. Gaudrault, o.p., Onzième session, p. $248-49$.

3.23 - Je pense, en troisième lieu, que l'on pourrait étendre à la société québécoise de cette époque, et sans doute aussi à la nôtre, ma troisième thèse, celle qui est relative à l'existence d'une formation réactionnelle. L'Académie thomiste nous aurait à cet égard légué un type de conscience. Ce type de conscience serait moins une conscience malheureuse qu'une conscience dissociée. L'Académie Saint-Thomas d'Aquin exigeait de ses membres qu'ils fussent originaux tout en demeurant soumis à la dictature, pour reprendre une expression de cardinal Villeneuve, d'une doctrine. Cette situation contradictoire où l'on requiert de quelqu'un la chose même qu'on lui retire les moyens de réaliser me paraît constituer l'écueil contre lequel s'est buté de façon récurrente notre communauté. Les exemples contemporains que je pourrais donner risqueraient de transformer cet exposé en un manifeste politique. Je laisse donc à votre imagination le soin de suppléer les exemples qui sont chargés politiquement et me contenterai d'alléguer un exemple pris dans le champ de la production intellectuelle. La production théorique et/ou artistique au Québec a toujours tenté de satisfaire à deux exigences qui, lorsqu'elles constituent une déontologie explicite, sont contradictoires. La requête qui est faite à notre production intellectuelle de réfléchir jusqu'à la complaisance les idiosyncrasies de la société québécoise et d'opérer de la sorte à la manière d'un exorcisme communautaire se redouble, de façon perverse, de la condition qui lui est assignée de "faire universel ". Or l'une et l'autre exigences reposent également sur un malentendu car elles portent sur des objets qui ne peuvent faire l'objet d'une volonté ou d'une décision de la part de l'artiste ou du théoricien. Il n'appartient pas plus à la production intellectuelle de choisir de réfléchir les particularités de son contexte d'origine que d'ambitionner de "faire universel ". La première de ces exigences constitue le présupposé de la production intellectuelle et la seconde se rapporte essentiellement à ses effets chez un récepteur éventuel ou à son résultat. La production théorique et/ou artistique est d'emblée et $a$ priori habitée par les tensions de son contexte d'origine et sa valeur 
d'universalité est quelque chose qui lui est conférée aprèr conp et a posteriori par ceux qui la reçoivent. La plus universelle des créatures de Shakespeare, Falstaff, apparaît dans les pièces qui ont pour objet le destin particulier des rois d'Angleterre.

3.24 - Il est enfin une dernière chose que nous connûmes au cours de ces séances de l'Académie canadienne Saint-Thomas d'Aquin. C'est la saveur doucereuse de l'abjection. Retrouvons-la brièvement en écoutant parler un avocat :

Ensemble de textes 29 :

L'entrée des lä̈ques dans l'Académie canadienne Saint-Thomas d'Aquin parut à maintes gens une audace. C'en est une autre que de les y faire parler. Les simples mortels dont je suis avaient-ils leur place dans cet aréopage où philosophies et docteurs se proposent de commenter les enseignements du docteur angélique? En faire des auditeurs, passe encore. Mais leur assigner un rôle actif, seul le besoin d'an. tithèse expliquerait pareille initiative.

A. Perrault, Première session, p. 141.

Il y a trente ans j’ai commencé à subir l'ascendant de l'esprit de Mgr L-A. Pâquet. Je n'ai jamais cherché à m'y soustraire. Chaque jour je remercie Dieu d'avoir placé sur ma route la lumière de cette intelligence, l'amitié dont il a bien voul u m'honorer. Un mot Mgr Pâquet est pour moi un ordre. Je lui ai voué obéissance.

(p. 143).

Mais pour que son cuvre ne soit pas néfaste ou vaine, vous apercevez à quelles conditions le laïque doit aussi collaborer à l'apostolat doctrinal de l'Église. Il ne saurait être le dirigeant. Son rôle est d'aider non de guider. Et son premier devoir est de connaître les prescriptions de l'autorité religieuse et d'y demeurer fidèle. D'un mot Mgr L-A. Pâquet résuma cette attitude imposée aux lä̈ques : "le zèle des lä̈ques doit se renfermer dans de justes limites de soumission de docilité et de déférence vis-à-vis l'autorité religieuse".

(p. 180). 
Prêchant l'absolu, les laïques vivent dans le relatif. Que de récifs! Les passes dangereuses sont éclairées par les feux fixes des encycliques papales. La lumière qui en descend permet-elle, chaque jour et pour chaque difficulté, de voir l'écueil et de l'éviter? Les circonstances posent constamment des problèmes dont la solution par le souverain pontif n'interviendra que plus tard. En attendant il faut agir, agir en esprit de simplicité, et de droiture. La règle de conduite ne sera-t-elle pas la soumission à la hiérarchie immédiate..?

On peut difficilement nier l'existence d'une certaine gêne à la lecture d'un texte comme celui qui vient d'être cité. Ce sentiment de gêne a dû aussi se manifester à la lecture d'autres textes que j'ai reproduits. J'aimerais à cet égard énoncer un double souhait, en terminant. Je souhaiterais d'abord que les réactions de toutes natures que ne manqueront sans doute pas de susciter les exemples que j'ai produits n'inhibent pas l'attention que l'on devrait apporter à l'évaluation de mes hypothèses. Ce sont elles qui constituent l'essentiel de ce texte, dont on aurait tort de croire qu'il est tourné vers un passé révolu. J'ai pu me rendre compte, suite à des publications antécédentes, que le thraumatisme que ravivait mes exemples empêchait que l'on prît un soin suffisant pour apprécier mes propositions. Ceci, malheureusement se produira encore et j'en pressens la façon. Plusieurs diront que j'ai délibérément choisi, dans la somme des textes que j'ai examinés, le pire et l'idoine. Je souhaite à cet égard que l'on prenne la peine de relire dans leur ensemble les textes qui ont constitué ma matière. On découvrira que bien loin d'avoir sélectionné le pire, qui aurait servi mon enttreprise, j'en ai passé et du moins bon. La session de 1945, pour prendre un exemple, affichait une communication de l'abbé J.Aderville Bureau de Québec, intitulée “ Les cryptorchides et les eunuques peuvent-ils contracter mariage?". Je laisse à l'appréciation du lecteur ce qu'un humour malveillant aurait pu tirer d'un tel programme.

Pourquoi, enfin, sonder les placards pour en retirer des squelettes? On pourrait évidemment revendiquer l'autonomie du projet théorique et contester les termes d'une métaphore qui veut se donner pour une question. Cette réponse est la plus juste mais elle présuppose, pour être entendue, une sérénité qu'il est difficile 
de reconnaître à ceux qui soulèvent cette question. Nous situant où ils se placent, nous répondrons donc: parce que beaucoup de ces squelettes dansent encore parmi nous, gainés d'une nouvelle chair.

\section{Deux remarques}

* L'une des critiques le plus souvent adressées à ceux qui, comme moi, situent avant tout leurs analyses au niveau du discours consiste justement à déplorer que l'analyse choisisse de se maintenir au niveau du discours, sans apparemment se soucier de rechercher les conditions d'ordre infrastructurel (" en dernière instance économiques") qui président à la production des discours et qui déterminent souvent leurs effets. L'énoncé de ce type de critique est suffisamment fréquent pour que l'on consacre quelques paragraphes à y répondre.

Je remarquerai d'abord que cette critique se présente la plupart du temps sous une double forme. Une première variante, que je qualifierai d'épistémologique, tient dans une série de propositions, souvent parfaitement recevables, qui affirment que l'analyse des discours constitue une entreprise régionale qui aurait intérêt à rapporter le discours à autre chose qu'à lui-même et qui a parfois en outre le tort de confondre la syntaxe des phrases avec les processus de l'histoire. Il est cependant une autre variante de cette critique, dont l'occurrence est plus fréquente, et que je pourrais indifféremment qualifier de militante, par rapport à son style, ou d'essentialiste, par égard à son contenu. Selon cette seconde variante, la critique procède de la manière suivante. On fait d'abord valoir de façon péremptoire le primat de l'économique ou celui de quelque autre instance fondamentale dans l'étude de tout phéno. mène qui est justiciable d'une histoire; on décrète ensuite que toute entreprise théorique qui n'est pas avant tout préoccupée de reconduire les phénomènes dont elle fait l'analyse à l'instance réputée ultime, et dont la lutte des classes constitue l'horizon familier, ne peut avoir qu'un objet non essentiel ; on juge enfin, en arguant à partir d'une certaine "urgence " d'effectuer quelques tâches théoriques privilégiées, que tout discours qui n'est pas fasciné par l'essentiel, tel qu'il a été défini, est équivalent à un nondiscours théorique. Qui parle d'autre chose que de ce qui a été reconnu comme essentiel ne dit rien.

Comme je l'ai dit plus haut, la forme épistémologique de la 
critique à laquelle je m'efforcerai maintenant de répondre recèle une part très assurée de vérité. La troisième section de mon article devrait à cet égard être complétée. La ligne qui sépare cependant cette forme heuristique de la critique de sa forme militante ou essentialiste m'apparaît cependant d'un tracé bien incertain, de telle sorte que l'épistémologie dérive trop souvent vers l'essentialisme. Voici maintenant ce que j'ai à dire par rapport à cette seconde variante, plus répandue, de la critique de l'analyse du discours. J'ai d'abord toujours été extrêmement frappé par la disproportion qui existe dans ce type de critique entre la part qui est faite à l'exigence et celle qui est faite à l'exemple. Les arguments qu'on nous sert m'apparaîtraient plus convaincants s'ils faisaient autre chose que ressasser un programme de recherche que d'autres auraient la charge de remplir; la généralité non dépassée des propositions qui ont jusqu'ici été produites par ceux-là mêmes qui réclament une sortie de la théorie des discours vers la matérialité de l'histoire n'est certes pas de nature à convaincre ceux qui ont peu d'habitude à l'effectuer de son opportunité. Il y va de la théorie comme du sacerdoce : on souhaiterait que la ferveur à pratiquer s'égalât au moins au zèle à convertir.

La requête qui nous est faite de rapporter les discours à leurs conditions matérielles de production tire une grande partie de son pouvoir d'interpellation de sa conformité avec une exigence prag. matique qui a reçu son brevet de naturalité et selon laquelle la pertinence d'une discipline et la validité de ses résultats peut se tirer de la réalité de ses objets. Le rabattement de l'abstrait sur le concret, ou du moins sur ce qui est décrété tel, est une opération qui nous apparaît aussi raisonnable que le rejet de l'évanescent au profit de ce qui est durable et agissant. Cette quête du "réellement réel " me semble à l'examen aussi abstraite que les métaphysiques qui lui ont donné naissance. Que l'on s'en réfère en effet à la science. Les problèmes qui sont soulevés par la réduction interthéorétique y sont abondamment discutés. Il est plusieurs linguistes qui, par exemple, pensent que le savoir linguistique absolu se produira comme une psychologie ; une psychologie fondamentale prendrait à son tour de façon croissante la forme d'une neurologie ou d'une névrologie; celles-ci s'achèveraient éventuellement dans une biologie se réduisant elle-même enfin, à travers les intermédiaires d'une biochimie et d'une bio-physique, à une physique. $\grave{A}$ n'en pas douter, ces programmes existent. Ils constituent cependant bien davantage 
des idées régulatrices qu'une invitation à abandonner la recherche à tous les niveaux médians au profit de celui en lequel ils devraient tous finir par se résoudre. Le concept même de réduction interthéorétique implique l'existence de la recherche à tous les niveaux de l'édifice : le niveau $A$, s'il cesse d'exister, ne pourrait être traduit dans le langage du niveau $B$ ou même mis en relation avec lui. C'est pourquoi on ne saurait penser, même si nous en venions à posséder toutes les règles d'une réduction interthéorétique, que la découverte de ces règles entraînerait l'abolition de toutes les disciplines au profit de celle qui a pour objet la réalité matérielle ultime de tous les objets du savoir. On imagine dès lors assez mal un physicien qui se ferait une carrière d'assister aux débats de ses collègues linguistes, psychologues ou biologistes en leur enjoignant plus ou moins péremptoirement d'abandonner leurs errements idéalistes au profit d'une théorie de l'instance ultime. On caricature à peine en disant que c'est cette sotte invitation qui est faite de façon réitérée aux chercheurs des sciences humaines, où il n'est aucune discipline qui n'ait considéré, sous la pression d'intervenants, de se redéfinir comme économie politique.

Je tenterai d'être explicite, au risque d'être mal entendu. Mon propos n'est pas de produire un plaidoyer en faveur de l'excarnation de la recherche en sciences humaines. Je refuse cependant de penser qu'un acquiescement, serait-il sans réserve, à des propositions aussi abstraites et aussi controversées que celles qui ambitionnent de nous désigner l'essentiel et l'ultime implique que l'on doive renoncer à poursuivre des recherches qui produisent des propositions vraies sous le prétexte qu'elles n'auraient pas la bonne fortune de se situer à l'intérieur du périmètre de ce qui a été posé comme l'essentiel. En une seule phrase : il est ruineux pour une politique de la recherche d'assimiler ce qui est pertinent à ce qui a été défini comme essentiel.

Pourquoi d'ailleurs nous mettrions-nous tous soudainement à rapporter les phénomènes que nous examinons à leurs conditions matérielles de production. Non seulement cela n'est-il pas requis mais, dans la mesure où la difficulté de ces recherches se laisse soupçonner de l'espoir qu'elles suscitent, on ne pourrait que mal augurer d'un recyclage improvisé et abrupt de tous les chercheurs qui travaillent dans le champ des sciences humaines. Que ceux-là qui jugent depuis longtemps qu'une telle reconduction est né- 
cessaire et qui ont dû produire la méthodologie qui permettra qu'on l'effectue avec rigueur reprennent le fardeau de l'analyse là où d'autres, moins clairvoyants, l'ont déposé et qu'ils s'emploient à le porter plus loin. Il n'est personne qui refusera d'être instruit par les résultats qu'ils obtiendront. Cette répartition des tâches m'apparait raisonnable et j'avouerai pour ma part, en terminant, n'avoir jamais compris pourquoi les partisans de l'établissement d'une jonction entre les objets des sciences humaines et leurs conditions matérielles de production répugnaient tant à devenir les partenaires d'une recherche diversifiée et si peu à vouloir s'en faire les contremaîtres.

** L'une des questions le plus souvent soulevées dans la discussion des propositions avancées par ce texte fut celle des rapports entre le concept d'orthodoxie et celui d'idéologie. Cette question est vaste et je ne prétends pas la traiter de façon exhaustive dans cette seconde remarque. Je me limiterai à présenter ce qui m'apparaît pour l'instant clairement. Je m'en rapporterai, pour ce qui est de la théorie de l'idéologie, à l'article, justement influent d'Althusser, intitulé "Idéologie et appareils idéologiques d'État" (La pensée, no 151, mai-juin 1970, p. 3-38). Je compte moins retenir la définition de l'idéologie que propose cet article (représentation du rapport imaginaire des individus à leurs conditions réelles d'existence, p. 24) que certaines des propositions d'Althusser sur le fonctionnement des appareils d'État. "Ce qui distingue les AEI (appareils idéologiques d'État) de l'Appareil (répressif) d'État, écrit Althusser à la p. 14, c'est la différence fondamentale suivante : l'Appareil répressif d'État " fonctionne à la violence ", alors que les Appareils idéologiques d'État fonctionnent "à l'idéologie " (souligné dans le texte). Bien sûr, Althusser a soin de préciser que cette distinction violente/idéologie réfère davantage à un fonctionnement prévalent des Appareils d'État qu'à des opérations exclusives. Il n'y a pas de pur appareil répressif, comme il n'y a pas de pur appareil idéologique; néanmoins les privilèges sont suffisamment marqués pour qu'Althusser nous parle d'un fonctionnement " massivement prévalent " à la répression violente ou à l'idéologie (p. 14).

C'est par rapport à cette distinction entre deux types de fonctionnement des appareils institutionnels que j'aimerais brièvement situer la notion d'orthodoxie. Il apparaît à première vue clair que 
notre notion d'orthodoxie réfère davantage au champ de l'idéologie qu'à celui de la répression violente. "Orthodoxe" est un prédicat qui qualifie habituellement les doctrines et qui peut en conséquence s'appliquer aussi aux idéologies. Sans contester cet aperçu de sens commun, j'aimerais cependant lui apporter la précision suivante. Ce que désigne proprement la notion d'orthodoxie, c'est l'intrusion d'une violence au sein de l'appareil où se produit et se diffuse le savoir pour assurer la persistance de son fonctionnement à l'idéologie. De ce point de vue, l'orthodoxie constituerait le point limite où un appareil qui fonctionne à l'idéologie doit être relayé par un appareil qui fonctionne à la violence (à la sanction) pour continuer d'opérer. Si l'on peut croire avec Althusser que l'idéologie n'a pas d'histoire, il est néanmoins permis de concevoir que son mode d'existence est cyclique, les différents moments de ce cycle étant constitués par la croissance et la dégénérescence progressives de son pouvoir d'interpellation. Située au terme du cycle, l'orthodoxie se définirait comme la forme dégénérée d'une idéologie, dont le pouvoir d'interpellation a tellement décru qu'il doit recourir à un pouvoir institutionnel répressif pour s'exercer encore. L'évolution de ce qu'Althusser désigne comme l'idéologie religieuse chrétienne (avant ce que l'on a appelé en suite de Nietzsche la "mort de Dieu ") ne démentirait pas l'existence du cycle dont nous avons tenté de décrire le dernier moment.

Département de Philosophie Université du Québec à Montréal 